\title{
Interdisziplinäre Lehre und Forschung für die Medienwirtschaft an der Fach- hochschule St. Pölten
}

\begin{abstract}
Die Medienwirtschaft hat sich in den letzten Dekaden tiefgreifend verändert. Die enorme Vielfalt an Kanälen und Plattformen, die Fragmentierung der Märkte, sinkende Erlöse und die zunehmende Konkurrenz durch globale Konzerne stellen die Medienunternehmen in Europa vor große Herausforderungen. Gleichzeitig eröffnen sich mit der Digitalisierung neue Möglichkeiten der Herstellung und Distribution von Content. Mit ihren datenbasierten Diensten werden die Medienunternehmen zu Innovationstreibern der digitalen Ökonomie.
\end{abstract}

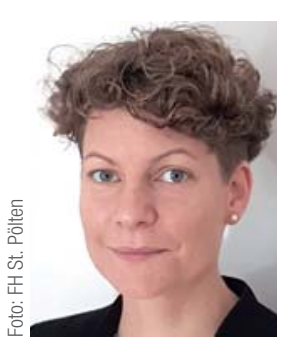

FH-Prof. Mag. (FH) Dr. Johanna Grüblbauer

Fachhochschule St. Pölten

Department Medien und Digitale Technologien Studiengangsleiterin Medienmanagement (BA)

johanna.grueblbauer@fhstp.ac.at

Schlüsselbegriffe: Medienmanagement I Technologie I Medienproduktion | Journalismus I Crossmedia

Der Medienschwerpunkt an der Fachhochschule St. Pölten

Die Fachhochschule St. Pölten schaut auf eine 25-jährige Geschichte der Lehre und Forschung im Medienbereich zurück. Durch die Bündelung aller Kräfte über die Grenzen der Disziplinen hinweg entsteht nun ein neues, einzigartiges Angebot, das die technischen, die betriebswirtschaftlichen und die kreativen Aspekte der Medienproduktion vereint, Synergien für die strategische Weiterentwicklung der Studiengänge schafft und damit einen wichtigen Beitrag zur Bewältigung der Herausforderungen in der Medienwirtschaft leistet.

Das medienbezogene Studienangebot an der Fachhochschule St. Pölten umfasst derzeit fünf Bachelorstudiengänge, sieben Masterstudiengänge und sieben Weiterbildungslehrgänge, die vom Medienmanagement über Medientechnik und Data Science bis hin zum Marketing alle Aspekte der digitalen Medienwelt berücksichtigen.

Das Institut für CreativelMedia/Technologies und die Forschungsgruppe Media Business

Am Institut für CreativelMedia/Technologies (IClM/T) forschen mehr als 30 wissenschaftliche Mitarbeiter*innen an der Schnittstelle von Technologie, Wirtschaft, Gesellschaft und Kunst. Ziel der Forschung ist es, zu einem tieferen Verständnis der Gestaltung, Produktion und Nutzung digitaler Medientechnologien beizutragen wie auch die Wechselwirkungen von Technologie und Gesellschaft zu analysieren. Im Zentrum steht dabei ein nutzer*innenzentrierter Ansatz, d.h. die Frage, wie Menschen mit den Technologien interagieren und welchen Nutzen sie daraus ziehen.

Das Institut bestand bislang aus den Forschungsgruppen Digital Technologies, Media Computing und Media Creation. Durch die Integration der Forschungsgruppe Media Business im Sommer 2020 wurde das Spektrum der Forschung um zentrale medienwirtschaftliche Fragestellungen erweitert. Die Forschungsgruppe hat es sich zur Aufgabe gemacht, zentrale Aspekte der digitalen Transformation der Medienwirtschaft zu dokumentieren, zu analysieren und zu evaluieren und damit eine Grundlage für unternehmerische und medienpolitische Entscheidungen zu schaffen. Die Forschungsthemen reichen dabei von den Auswirkungen der Digitalisierung auf die Leistungserstellungs- und Erlösmodelle von Medienunternehmen, über Fragen der Governance von digitalen Plattformen bis hin zu den gesellschaftlichen und ethischen Folgen von Algorithmen und Big Data in der Medienwirtschaft.

Das Team des ICIM/T, das aus Expert*innen unterschiedlichster Disziplinen zusammengesetzt ist, bietet sowohl 
theoretisch fundierte Grundlagenforschung als auch praxisorientierte Dienstleistungen für Unternehmen, zivilgesellschaftliche Akteur*innen und die öffentliche Verwaltung. Das Portfolio an Leistungen umfasst Forschung und Entwicklung von der Problemanalyse über das Design technischer Lösungen bis hin zur Implementierung von Prototypen. Darüber hinaus verfügen die Mitarbeiter*innen über profunde Expertise im Bereich empirischer Forschungsmethoden und Usability Research. Dazu gehören Branchenanalysen, Evaluationen, Experimente, statistische Sekundäranalysen, Online-Befragungen, Netzwerkanalysen, Social Media Analysen, Webanalytics, Fokusgruppen, Expertinnengespräche, Leitfadeninterviews, quantitative und qualitative Inhaltsanalysen, apparative Methoden wie Eyetracking und Biofeedback (EDA) und vieles mehr.

Informationen über aktuelle Projekte des Instituts finden Sie unter https://icmt.fhstp.ac.at/.

\section{Das neue Curriculum des Studiengangs Medienmanagement}

Im Zuge der Eingliederung des Studiengangs in das Department Medien und digitale Technologien wurde das Curriculum überarbeitet, um den Anforderungen einer digitalen Medienwelt gerecht zu werden. Das Entwicklungsteam des neuen Curriculums ging dabei von einigen zentralen Prämissen aus:

Eine zeitgemäße Medienmanagementausbildung

- zielt auf ein crossmediales Mindset ab, das sich weniger an Mediengattungen und mehr an Stories orientiert,

- schafft ein Qualitätsbewusstsein für alle ContentArten (News, Entertainment und Inspiration) mit ihren unterschiedlichen Zielsetzungen,

- reflektiert die ethischen, politischen und kulturellen Rahmenbedingungen der Medienproduktion in einem vereinten Europa,

- verbindet das Handwerk der Medienproduktion mit dem Wissen um Kosten und Erlösmodelle,

- vermittelt Technologie-Know-how und ein grundlegendes Verständnis für die digitale Transformation,

- $\quad$ sensibilisiert für das strategische Management von Innovations- und Change-Prozessen.

Das dreijährige Studium integriert zudem ein verpflichtendes Berufspraktikum und ein Projektsemester, das nicht nur Raum für interdisziplinäre Projekte, sondern auch die Gele-

\section{Abb. 1: Module}

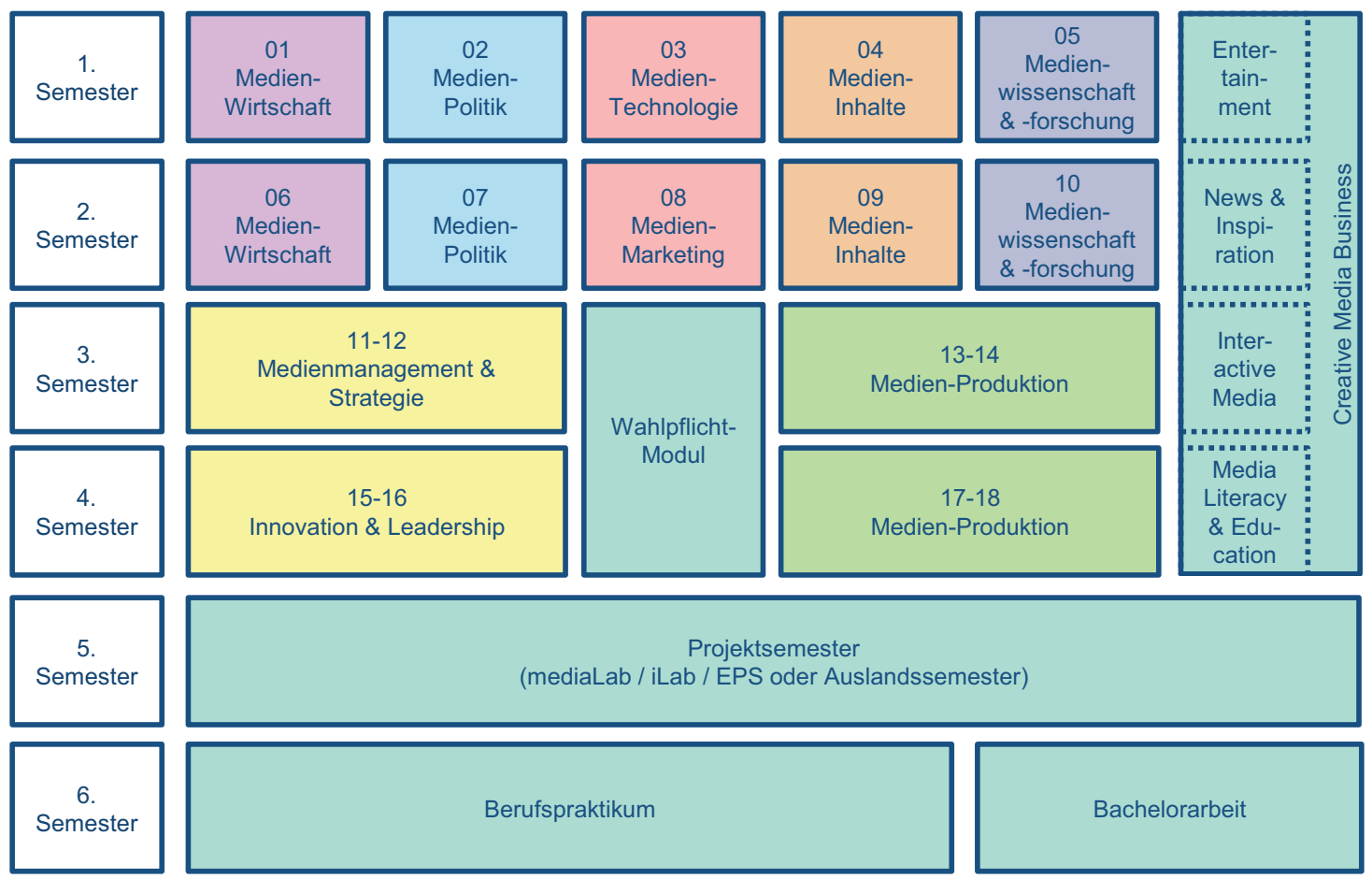




\section{Abb. 2: Schwerpunkte BMM}

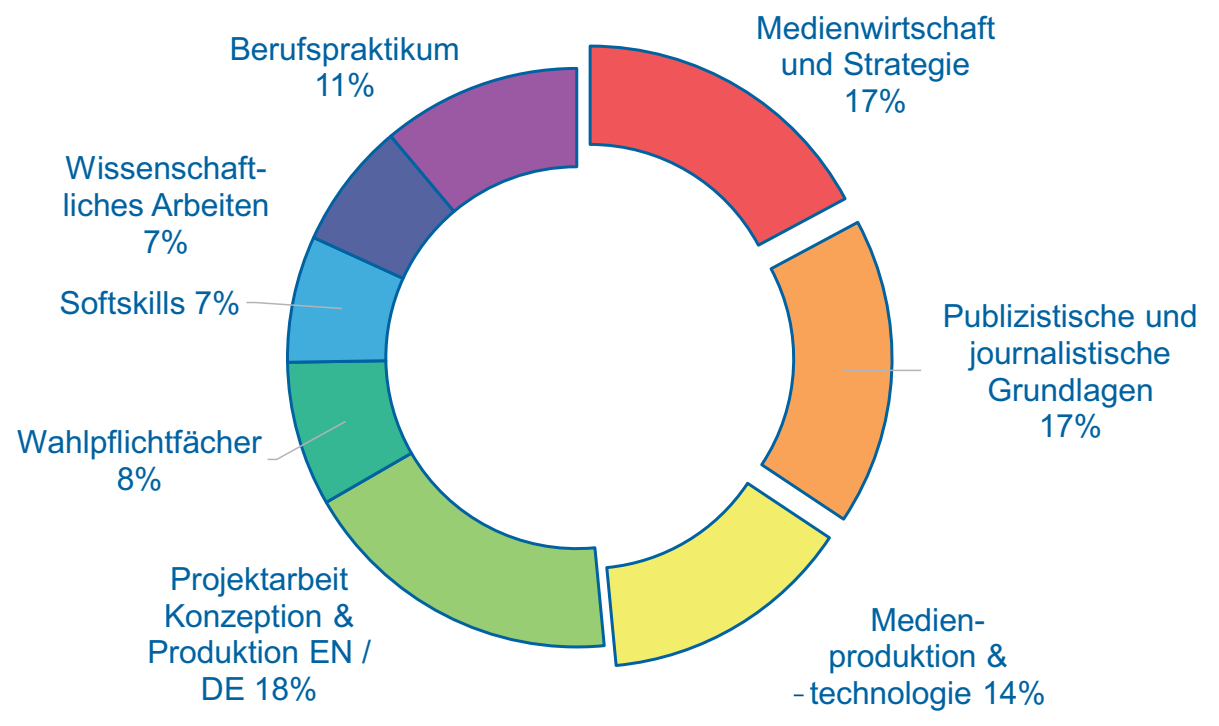

Quelle: FH St. Pölten

genheit für einen Auslandsaufenthalt bietet. Abbildungen 1 und 2 geben einen Überblick über das Curriculum ab dem WS 2021

Ein besonderes Augenmerk wurde auf die interdisziplinäre Vernetzung von betriebswirtschaftlichem Denken, technologischem Wissen und journalistischer, gestalterischer Praxis gelegt. Dazu wurden in das Curriculum spezielle Formate und "Windows of Opportunity" eingebaut. So bietet etwa die zweiwöchige Blockveranstaltung Creative Media Business am Ende jedes Semesters den Studierenden die Möglichkeit, konzentriert die während des Semesters gelernten Fertigkeiten in ein Projekt zu integrieren. Dazu werden die gelernten Inhalte des jeweiligen Semesters aus Perspektive bestimmter Mediennutzungsszenarien bzw. Value Pro- positions projektbasiert verknüpft und angewendet. Zudem haben die Studierenden die Möglichkeit, anhand von so genannten ePortfolios inre individuellen Professional Skills zu reflektieren.

Eine weitere Möglichkeit des interdisziplinären Austauschs bietet das Projektsemester, in dem in kleinen Teams studiengangsübergreifend an konkreten Geschäftsideen und technischen Applikationen gearbeitet wird. Ein Team von Expert*innen aus Wissenschaft und Praxis begleitet die Entwicklung bis hin zur Marktreife.

Weitere Informationen zum Studiengang unter https:// www.fhstp.ac.at/bmm oder bei der Studiengangsleitung.

\section{Impressum}

$\begin{array}{ll}\text { Herausgeber: } & \text { Schriftleitung: } \\ \text { Prof. Dr. Martin Gläser, } & \text { Prof. Dr. Martin Gläser (verantwortlich) } \\ \text { Hochschule der Medien Stuttgart } & \text { Hochschule der Medien, } \\ \begin{array}{l}\text { Prof. Dr. Georgios Gounalakis. } \\ \text { Philipps-Universität Marburg }\end{array} & \text { Nobelstraße 10, } 70569 \text { Stuttgart } \\ \text { Prof. Dr. Thomas Hess, } & \text { Verlag: } \\ \text { Ludwig-Maximilians-Universität München } & \text { New Business Verlag GmbH \& Co. KG } \\ \text { Prof. Dr. Frank Lobigs, } & \text { Nebendahlstraße 16. 22041 Hamburg } \\ \text { Technische Universität Dortmund } & \text { Tel.: 040 - 609 009-0 } \\ \text { Prof. Dr. Christoph Neuberger. } & \text { Fax: 040 - 609 009-15 } \\ \text { Frei Universität Berllin } & \text { e-Mail: infolanew-business.de } \\ \text { Prof. Dr. Insa Sjurts, } & \\ \text { Hamburg School of Business Administration } & \text { Verleger: Peter Strahlendorf } \\ & \text { Kfm. Geschäftsführung: } \\ & \text { Antje-Betina Weidlich-Strahlendorf } \\ & \text { Projektbetreuung: Natascha Przegendza } \\ & \text { Layout: Anne Allert, Antje Baustian }\end{array}$

Vertrieb: Angelika Schmidt

Anzeigen: Jens Jansen (verantwortlich)

Anzeigendisposition: Birgit Weselmann

Bankverbindung:

BAN: DE74200505501217131323

BIC/SWIFT: HASPDEHHXXX

IBAN: DE07200400000482282100

BIC/SWIFT: COBADEFFXXX

Druck und Lithos:

Lehmann Offsetdruck und Verlag GmbH

Gutenbergring 39, 22848 Nordersted

Bezugsbedingungen:

Jahresabonnement 78,- Euro (zzgl. Versand und USt.).

Studentenabonnement 49,- Euro (zzgl. Versand und USt.).
Das Abonnement verlängert sich automatisch um ein Jahr, wenn es nicht mit einer Frist von vier Wochen zum Ende des Bezugszeitraumes schriftlich gekündigt wird.

Erscheinungsweise: 4-mal jährlich

Namentlich gekennzeichnete Artikel müssen nicht die Meinung der Herausgeber/Redaktion wiedergeben. Un verlangt eingesandte Manuskripte - für die keine Haftung übernommen wird - gelten als Veröffentlichungsvorschlag zu den Bedingungen des Verlages. Es werden nur unveröffentlichte Orginalarbeiten angenommen. Die Verfasser erklären sich mit einer nicht sinnentstellenden redaktionellen Bearbeitung einverstanden. 